\title{
Integrated Display and Environmental Awareness System - System Architecture Definition
}

\author{
Ondrej Doule, Ph.D. ${ }^{1}$ \\ Florida Institute of Technology, School of Human-Centered Design, Innovation and Art, Melbourne, FL, 32901, \\ USA \\ David Miranda ${ }^{2} \&$ Jake Hochstadt $^{3}$ \\ NASA Kennedy Space Center, FL, 32899, USA
}

\begin{abstract}
The Integrated Display and Environmental Awareness System (IDEAS) is an interdisciplinary team project focusing on the development of a wearable computer and Head Mounted Display (HMD) based on Commercial-Off-The-Shelf (COTS) components for the specific application and needs of NASA technicians, engineers and astronauts. Wearable computers are on the verge of utilization trials in daily life as well as industrial environments. The first civil and COTS wearable head mounted display systems were introduced just a few years ago and they probed not only technology readiness in terms of performance, endurance, miniaturization, operability and usefulness but also maturity of practice in perspective of a socio-technical context. Although the main technical hurdles such as mass and power were addressed as improvements on the technical side, the usefulness, practicality and social acceptance were often noted on the side of a broad variety of humans' operations. In other words, although the technology made a giant leap, its use and efficiency still looks for the sweet spot. The first IDEAS project started in January 2015 and was concluded in January 2017. The project identified current COTS systems' capability at minimum cost and maximum applicability and brought about important strategic concepts that will serve further IDEASlike system development.
\end{abstract}

\section{Nomenclature}

$\begin{array}{ll}A R & =\text { Augmented Reality } \\ C F A & =\text { Cognitive Function Analysis } \\ C O T S & =\text { Commercial Off The Shelf } \\ G U I & =\text { Graphical user Interface } \\ H M D & =\text { Head Mounted Display or Helmet Mounted Display } \\ H S I & =\text { Human-System Integration } \\ I D E A S & =\text { Integrated Display and Environmental Awareness System } \\ P M & =\text { Project Management } \\ V R & =\text { Virtual Reality }\end{array}$

\section{Introduction}

\footnotetext{
A UGMENTED or virtual reality (AR/VR) head mounted systems were for a very long time proprietary to military or special applications. They were developed to provide faster access or more precise information to critical information that is usually provided by head down displays. The current availability of HMDs in the gaming,
}

\footnotetext{
1 Assistant Professor, School of Human-Centered Design, Innovation and Art, 150 W University Blvd., 32901 Melbourne, FL, AIAA Regular Member.

${ }^{2}$ AST Technical Engineer Operations Management, Operations Integration \& Advanced Planning, Mail Stop: LX$\mathrm{O} 3$.

${ }^{3}$ AST Software Systems Computer Engineer, Applications Engineering and Operations, Mail Stop: IT-C2
} 
fitness, healthcare, industrial training and simulations industries is significant. HMDs are slowly being introduced in production lines, inspection scenarios and a variety of industrial settings.

\section{A. HMD benefits from a theoretical use perspective:}

Several industrial projects represent operators and users interest in HMD AR/VR systems:

- Experimental use of HMDs within the ARVIKA project led by Siemens that executed research on wearable systems providing augmented reality data experimentally. The results proved that there was no suitable technology for HMD in 2000 due to their bulkiness, size, optics or overall performance. ${ }^{1}$

- HMDs were tested in scenarios and hardware for firefighters who require enhanced timely situational awareness during the rescue missions. Paris Fire Brigade training center and Fraunhofer Institute for Applied Information Technology tested HMD (head down) inside the firefighter's mask that provided additional information about the team position and status, ambient temperature, heartrate, oxygen as well as navigation to the building exit. ${ }^{2}$

- HMDs area essential part of pilots' navigation and awareness system in Air Warfare. Integrated with helmet they can provide significant increase in speed and precision in combat and high g-load. ${ }^{3}$

There are several reasons or potential benefits for use of AR/VR HMDs in general work or home environments that justify development costs for industry:

- Enhancement of activity efficiency through precise (localized, timed, supervised) procedural guidance, recording and further possibility of system and human learning / optimization

- Enhancement of human safety thanks to higher process control, process clarity, activity monitoring, environmental monitoring and warnings directly "on eye"

- Enhancement of affordances of human activity thanks to availability of virtual tools and components supporting reality or virtual reality immersion

- Enhancement and provision of instant or real time information processing thanks to wearable or portable computers and sensors that can analyze, sense, perceive, interpret and model information according to user needs or environmental settings allowing for more precise or correct decision making (philosophy derived from aviation using Mica Endsley model is applicable also in terrestrial settings using personal HMD or any type of computer operated cockpit) ${ }^{4}$.

\section{B. HMD drawbacks and challenges based on experience with existing hardware:}

The drawbacks of HMD relevant technology were in their mass, bulkiness, ergonomic discomfort (e.g., not sufficiently adjustable wearable system, sensors placement problem or balancing issues) ${ }^{5}$, tracking systems accuracy, display resolution and luminosity, and field of view that are balanced against usefulness and operability within personal comfort requirements. Probably the most advanced generally usable device in the HMD arena was the Google HUD Glass (further in the text referred to as "Glass"). Its high level of integration and extremely low mass allowed for long duration usage. The drawbacks such as battery lifetime, luminescence of the display and system control were temporarily overcome by its ease of wearability and solid performance. Still, the Glass kept looking for its application as a unique but generic and hence often only average applicability device. Nonetheless, Glass was the most user oriented HMD until now (beginning of 2017) but its "generic-use design" " made it difficult for serious application in industry or home. Glass is a technological breakthrough with very well thought through ergonomics but it lacks on the functional side. It seems as the engineering and hard ergonomics did an excellent job focusing on minimum mass, maximum power, display contrast, while functional (the understanding of use how the Glass should be used in practical scenarios) analysts were not fully admitted to the design. What Glass was missing in its early design process was not the traditional personas methodology used in product or display design but a very thorough Cognitive Function Analysis (CFA) method, used in aerospace Human-System Integration (HSI) that guides identification of functions and their human - system allocation. So it was observed using Glass from an HSI perspective is that the technological components were on a much higher level than their usage and applicability. This imbalance is related to a system architecture that might have lacked function analyses.

\footnotetext{
${ }^{4}$ Designed to be used in very broad types of environments and scenarios
} 


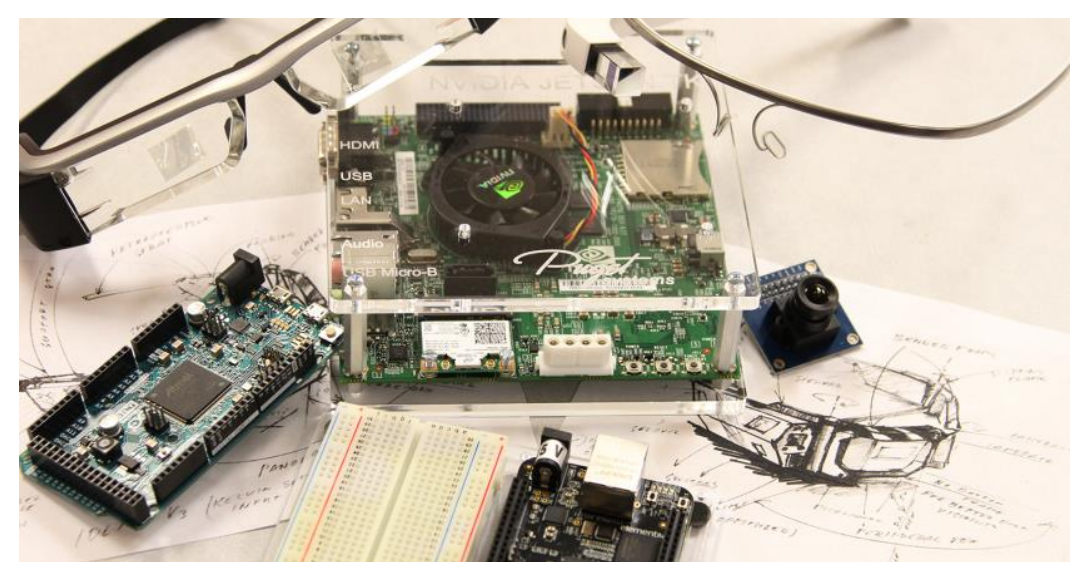

Figure 1: IDEAS early development environment, existing HMDs / smart glasses, development boards and sketches.

Since the Glass Explorer Edition launched in 2013 to only selected users, there has not been (to date January 2017) a more user friendly technological supplement on the market although many COTS wearable systems have been launched. Nevertheless, the technology and maturity of users evolved significantly and Glass brought many ideas about enhancement of humans' situational awareness. It also sparked the IDEAS project that identified specific functions, by knowing its users and environment that were given by its National Aeronautics and Space Administration (NASA) Kennedy Space Center (KSC) and HSF mission context. The IDEAS project narrowed down the HMD applicability and thus filled the gap that e.g., the Glass struggled with as a potential mass product due to its wide release and applicability.

\section{Methodology - IDEAS design and organizational methods}

The IDEAS team implemented many Human-Centered Design and Agile Management methodologies in order to understand the complexities of the NASA context, scenarios and user needs. Following these methods helped the team decode the design requirements and set initial priorities towards the design drivers.

\section{A. Product Research \& Innovation}

- Cognitive Function Analysis ${ }^{6}$ - A process that supported abstract understanding of functions, scenarios, tasks and derived activities and their allocation

- Scenario based concepts generation - Designer provides conceptual graphics and various scenarios while the team of experts provides feedback during and after the concepts generation. This method enabled the whole team to imagine how the system could physically look and how it could work in the field supporting the project architecture definition (Figure 2).

- Personas - Scenario based characters were defined and derived based on positions in organization related tasks. They were developed to understand required functions and features of the device

- Interviews - A number of potential users were interviewed and consulted $(25+)$ from different job positions and data was collected which contributed to the refining of the Personas and supported evolution of the concepts generated by the hardware and software teams (see Figure 3, Figure 4).

- Usability tests - These tests were incorporated in very early stages to understand the ergonomics of the system using COTS components, when the first functional system was in place. Usability tests were also performed on existing fully integrated COTS solutions such as wearable computers with HUD Epson Moverio, Microsoft HoloLens, Google Glass etc.

- Prototyping - Team utilized well developed NASA KSC prototyping infrastructure for development and testing of software, electronic and mechanical components and prototypes.

Copyright ( 2017 by Ondrej Doule, Ph.D., David Miranda and Jake Hochstadt

Published by the American Institute of Aeronautics and Astronautics, Inc., with permission. 


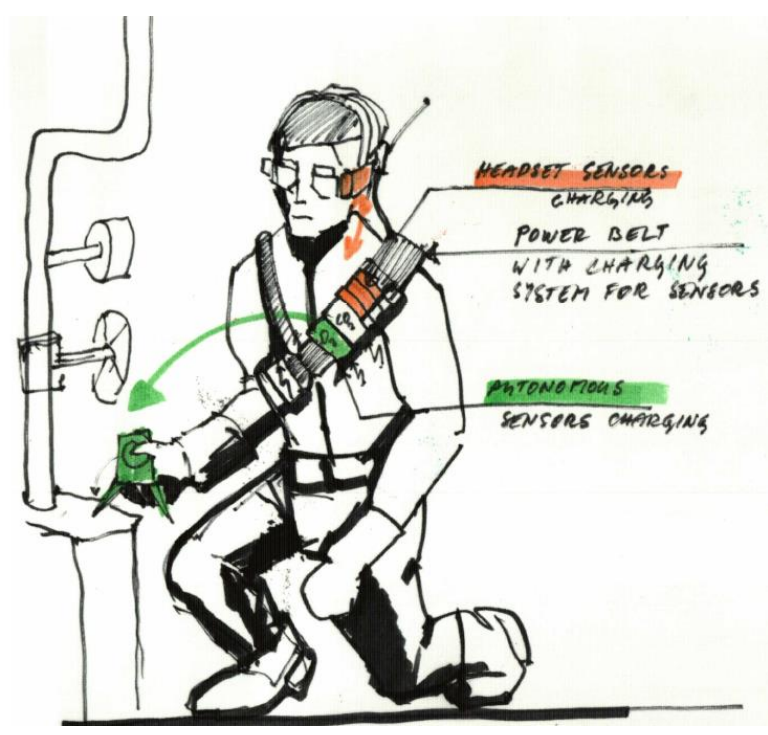

Figure 2: One of the considered scenarios sketch provides insight into possible HW/SW setup.

\section{SPECIAL APPAREL (PPE)}

\begin{tabular}{l|l}
\hline A & Gloves \\
B & Eye protection \\
C & Hearing protection \\
D & Hard hat \\
E & Hazmat suit \\
F & Jump suit \\
G & Respiratory protection
\end{tabular}

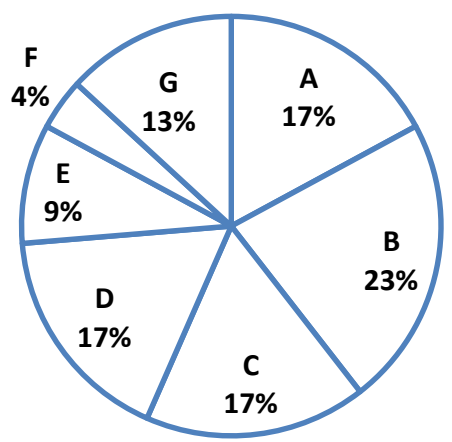

\section{REQUIRED TOOLS}

\begin{tabular}{c|l}
\hline H & Computer/Laptop/Tablet \\
I & Manuals documents \\
J & Cellphone \\
K & Radio transmitter \\
L & Video transmitter \\
M & Measurement tools \\
N & Pencil \& paper \\
O & Other hand tools
\end{tabular}

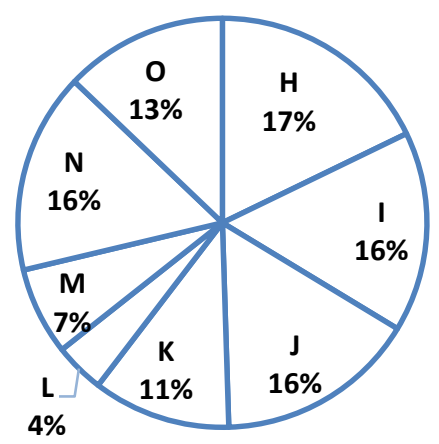

Figure 3: Interview-based data requirements regarding users' activities, tools and equipment. 
ENVIRONMENTAL HAZARDS

\begin{tabular}{l|l}
\hline A & Explosives \\
B & Compressed gasses \\
C & Flammable liquids \\
D & Flammable solids \\
E & Oxidizers \\
F & Poisons \\
G & Radioactive materials \\
H & Corrosive liquids \\
I & Environmental hazards
\end{tabular}

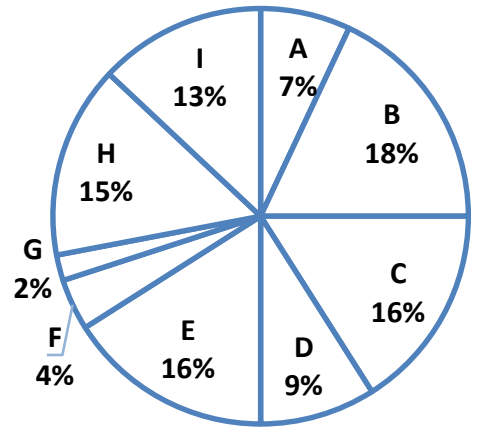

\section{WORK ENVIRONMENT}

\begin{tabular}{c|l} 
J & Noisy - verbal communication is difficult \\
K & Quiet \\
L & Full hands during work \\
M & Free hands during work \\
N & Low-normal light levels \\
O & Very bright environment \\
P & Info at work site available/ easy to use \\
Q & Info at work site not easily available/ easy to use
\end{tabular}

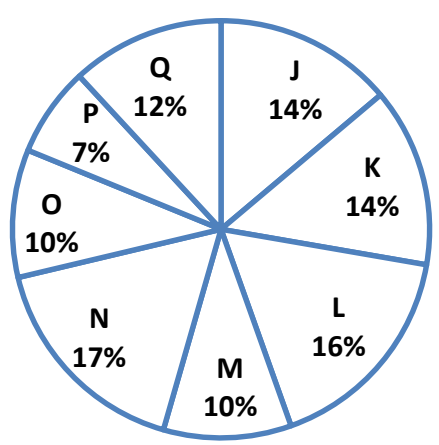

Figure 4: Interview-based data requirements regarding hazards and general work environment properties.

\section{B. Organization}

The team was coordinated in an agile project management manner. The project structure was based on the SCRUM methodology, which meant that every 9-10 project days a review with major stakeholders took place, who provided invaluable feedback and steering of the project goals and resources. The project was tracked, planned and coordinated using online platforms JIRA for task management and Confluence for data archive. SCRUM, widely used in software engineering, proved to be useful only to some extent in IDEAS product development. SCRUM is a difficult methodology to be used in an experimental project like IDEAS. Framing the creative process when developing the architecture or a completely new concept within the descriptive SCRUM could not be easily performed. SCRUM required: i.e. description of the creative process in advance in order to plan for it or schedule resources and project component links and outcomes including workforce. Although this PM method is considered agile, even this framework cannot capture emergent creative processes and hence some parts of the design process were created in an agile manner outside the SCRUM process without their record in stories or tasks e.g., unpredictable 25 iterations of a joint that had only one iteration / task registered in JIRA. SCRUM served well for engineering processes but not for the up-front conceptualization that had to be done before system engineering approach was started. Administrative requirement of breaking down the concept phase into sub tasks proved to be ineffective since the systematic division of the task on project managerial side would be ineffective time wise and technically impossible due to heuristic approach of the creative design process.

Although the team officially implemented the agile project planning and management approach and the design and development process has always been subject to discussion based on team members or stakeholders challenges, it has been often difficult to apply the agile approach within the NASA organization when reaching out for required resources. This paradox, when the team endorsed by the organization employs the agile processes is obstructed by the same organization to execute the agile approach, was never resolved. The experience of an agile team working in a non-agile environment has brought many slowdowns and inefficiencies that had to be dealt with extra effort on the design team side e.g., additional load to PM and team members who had to re-organize their tasks and modify the planned project chronology, to be able to move forward. The agility of the design team was also challenged by the 
fact that team members had to split their work effort across many diverse projects - i.e. not only working on the IDEAS project. In practice this meant that no single team member has been working on the project full time but often less or equal to 50 percent which was additionally broken in a non-consecutive flow of workdays. The obvious organizational problem of a non-agile upper organizational framework, limited work effort available and nonconsecutive workdays had to be mitigated, again, by the working team members themselves.

All these organizational specificities were amplified by unpredictably fast development in the domain of wearable computers and HMDs. A number of large corporations are investing in HMD systems and the COTS components IDEAS team relied on were simply evolving faster than the team could implement them mostly due to the organizational issues. On the one hand this phenomenon could appear positive as we are still looking into unevolved domain of wearable computers that are not mainstream and thus the team was hoping to get its hands on the best possible components. On the other hand, once the team found a suitable component there was a serious risk that the part would be discontinued or unsupported before the project ended. And this indeed happened. The team had to be very cautious about the parts and components to be employed especially from the future maintenance perspective.

The team was organized in three disciplinary and one inter/cross disciplinary team. The User Experience team was in charge of the concept definition while the hardware and software teams performed research and development on COTS and applicable integration methods. The integration team was composed of members of the three teams and assured compatibility of disciplinary approaches and their interoperability within the project's final product. Its competences (functions) were derived and dependent on experience with development of similar products.

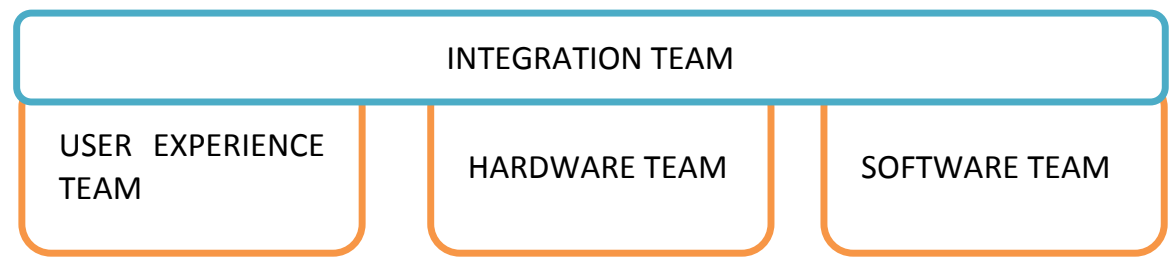

Figure 5: Team's organizational structure.

\section{System Design Requirements}

The design requirements were identified during the first quarter of the project development. The requirements were supported by interviews with the real users at NASA KSC (see Figure 3, Figure 4). All requirements were utilized for design of a comprehensive system architecture framework and scenarios. The long list of requirements was further prioritized and only the highest priority requirements were considered for the actual integrated hardware and software prototype using COTS components.

The main areas of interest and selected design requirements:

I. $\quad$ Primary goal Overall Requirements

$\circ$ Enhancement of user's awareness about the surrounding environmental hazards towards mitigation of user's risks associated with his/her task execution

- Faster tasks execution, minimum system mass, customizable ergonomics, procedural precision enhancement, guidance and navigation capability

II. Environmental Requirements

- Wearability in NASA KSC ground and high-rise operations environment

- System endurance to nominal wear/tear/impact, wild life safe, wearable while using PPE

- Software and data format compatibility with existing NASA databases

III. Operational Requirements

$\circ$ Long duration (4h) autonomous system (power/thermal) wearability (mass/ergonomics, SCAPE suit, space suit) untethered

- User's requirements on vision enhancement rather than blockage - i.e. no blocking of peripheral vision, no interference with user's prescription glasses, quick ergonomic adjustment

- Wearable system parts allow personal glasses, hardhat, HazMat, SCAPE or Space suit

Copyright $\odot 2017$ by Ondrej Doule, Ph.D., David Miranda and Jake Hochstadt Published by the American Institute of Aeronautics and Astronautics, Inc., with permission. 
AIAA Space Forum 2017, September 12-14, Orlando, FL

- Optional use of environmental safety sensors $\left(\mathrm{H}, \mathrm{O}_{2}, \mathrm{CO}_{2}, \mathrm{~N}_{2} \mathrm{H}_{4}, \mathrm{MMH}, \mathrm{N}_{2} \mathrm{O}_{4}, \mathrm{He}, \mathrm{NH}_{3}\right.$, gas pressure differentials, cryogens, hydraulic fluids, solvents, humidity, acoustic levels)

- Modularity - functions and systems de/mounting based on competences and needs

IV. Safety requirements

$\circ \quad$ Mitigation of interference with other tools

- Intuitive systems control operable in gloves

V. Communication Requirements

- Instant A/V, POV, remote sensor cameras

- Console and admin operators may select any view for control and supervision

- Remote control of cameras and sensors and data handling by second party / console operator

- Communication capabilities available in underground and Radio restricted areas

\section{Concept generation, scenario based design}

The generation of a variety of operational concepts and scenarios enabled quick decomposition of the users' environment complexity. The team conducted a number of interviews and discussions with NASA personnel to understand user needs as well as performed analyses of security, hardware and software infrastructure sought for emergent requirements through the creation of integrated concepts and scenarios generation (see Figure 6, Figure 7).

The main use scenarios at KSC correspond to operation of the space port, development and maintenance of infrastructure and transport of launcher systems (Figure 6). Space applications for astronauts during IVA or EVA were considered as well (Figure 7).

Five different user categories (roles) were created (Field Technician, Remote Operator, Quality Assurance personnel, Move Team and Astronauts) due to the difference of use and requirements on wearability, system endurance and difference in PPE. While the outdoors technicians often require a HMD compatible with PPE and special environmental requirements (e.g., radio-free areas, bright or dark conditions) most of the time a console operator or engineer is working in clean office environments with full access to technical infrastructure and the ability to regulate environmental properties. The IDEAS system for astronauts has to meet additional requirements on thermal, electrical and additional safety requirements due to the level of life-criticality of the space suit environment and sub-systems.

Copyright ( 2017 by Ondrej Doule, Ph.D., David Miranda and Jake Hochstadt Published by the American Institute of Aeronautics and Astronautics, Inc., with permission. 
IDEAS | USER components configuration

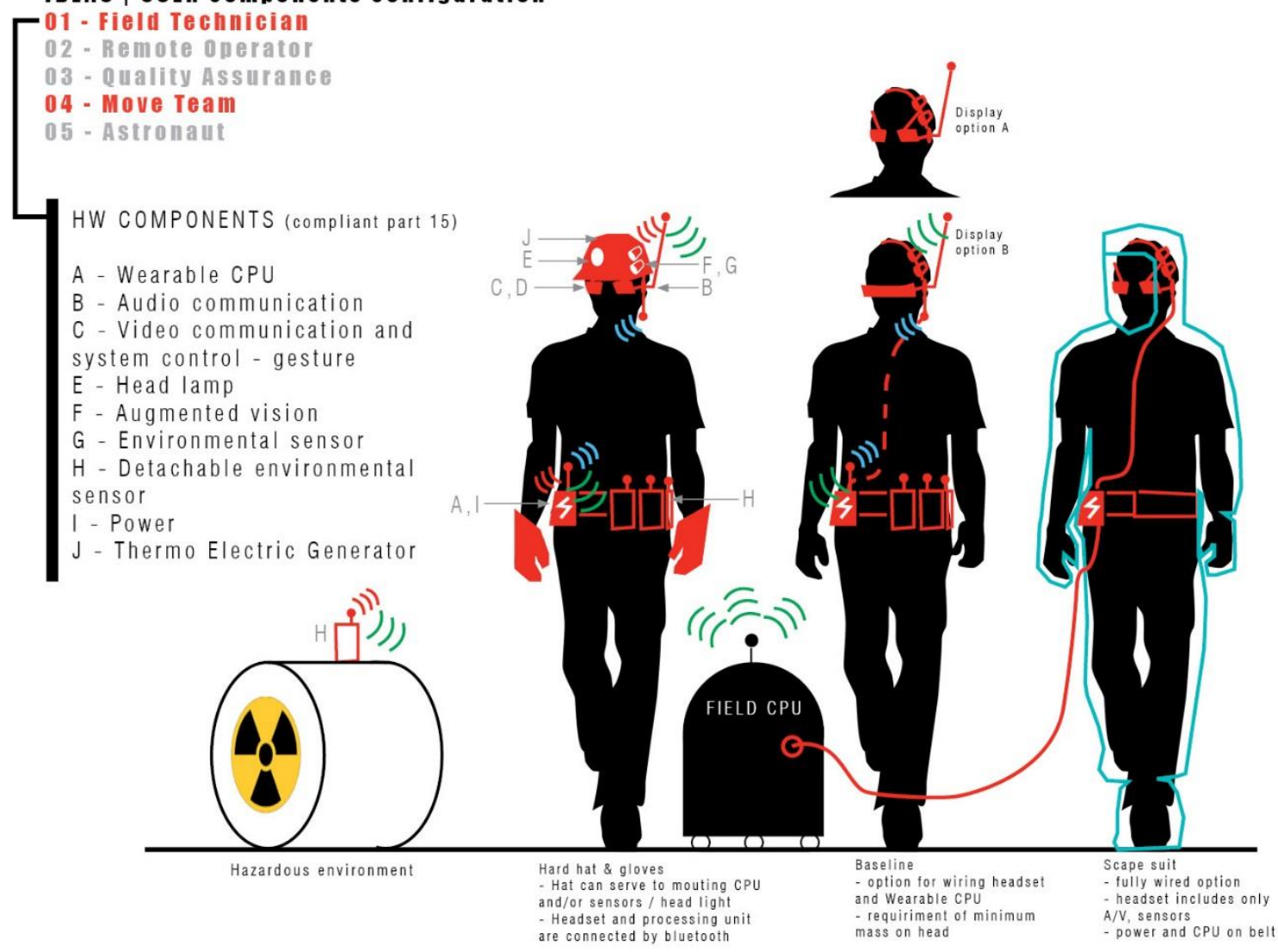

Figure 6: Analysis of possible use scenarios and wearability configurations based on functions and activity analyses. 
IDEAS | USER components configuration

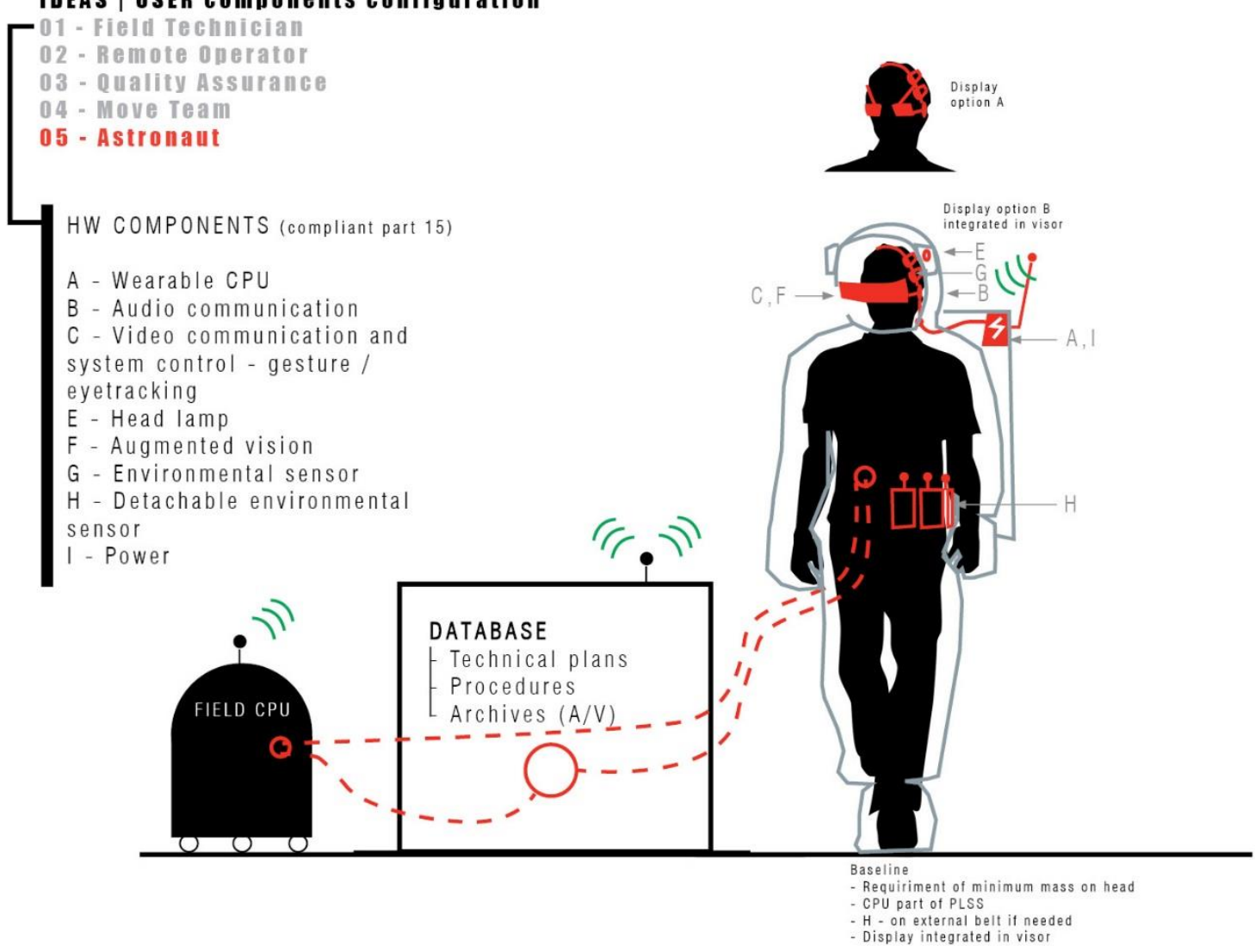

Figure 7: Scheme of the IDEAS system for astronaut's application.

Apart from the A/V communication, (i.e., possibility to send POV of self or other astronauts, communication with astronauts during space walk or IVA, and communicate with astronauts inside spaceship or station and be able to communicate with ground control and receive instructions) the astronaut looks for quickly reachable real time information about her/his life support quality and consumables duration and tools that help in comprehending and documenting the mission situation.

Based on the above five user categories the design requirements were updated. The variability in user requirements has put forth a requirement on incremental modular composition of the system based on individual needs. The proposed system architecture is depicted on Figure 8.

\section{Proposed system architecture}

The goal of the IDEAS team was to enhance the situational awareness of the end-user in a complex system such as KSC that deals with numerous life threatening events and hazards. The system architecture thus had to comply with existing data systems, communication channels, procedures and security requirements. The users were identified as field users (technicians, auditors, astronauts) and indoor operators (console operators, managers, designers, analysts etc.). The proposed architecture (see Figure 8) considered creation of new team intranets that are connected to the main network individually as clusters while allowing real-time communication using voice, image, video, archiving. 


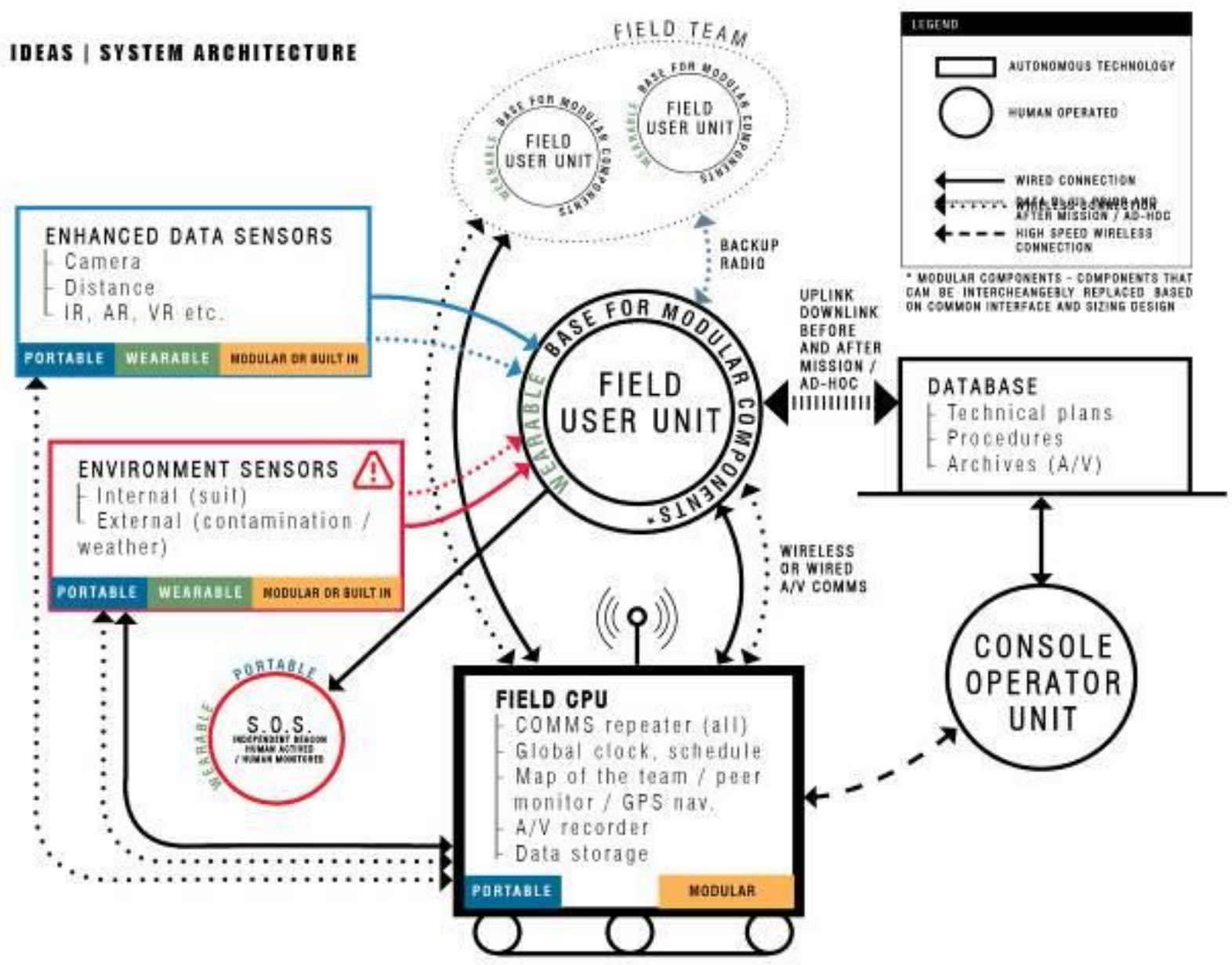

Figure 8: Initial proposed IDEAS system architecture for multiple users and automated unit.

\section{Proposed software architecture}

The dependence on the COTS components involved decisions about software platforms that would form the IDEAS operating system backbone.

The Android operating system was chosen due to the possibility of its use on a variety of hardware components and platforms (tablets/cellphones are broadly used and have high level of maturity of practice) rather than development of proprietary code or embedded Linux or even browser applicable interfaces. The web browser option had been also considered but abandoned due to the higher complexity of the system required in comparison to a pure Android platform with higher risks towards stability and computing requirements.

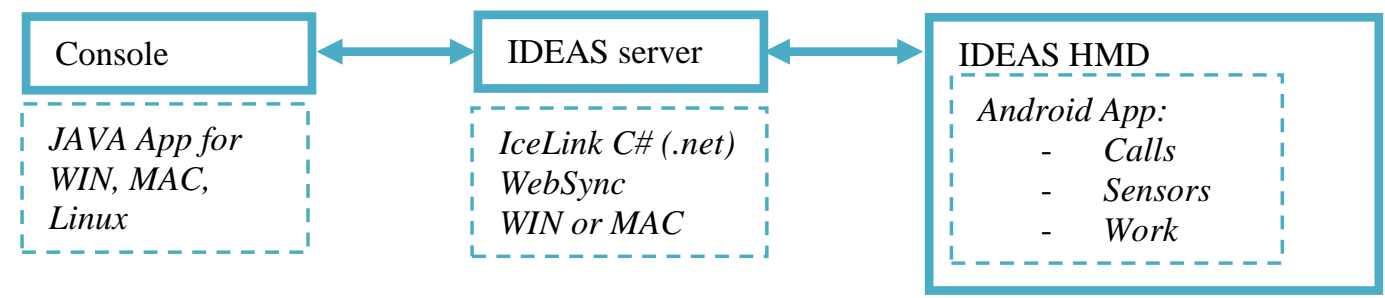

Copyright ( 2017 by Ondrej Doule, Ph.D., David Miranda and Jake Hochstadt Published by the American Institute of Aeronautics and Astronautics, Inc., with permission. 
The IDEAS server is based on two major components:

- IceLink - component that provides $\mathrm{A} / \mathrm{V}$ communication and file transfer data sharing enabling multiple users communications that are managed without accessing the central server

- WebSync - component enables and supports connectivity, telemetry, and messaging of IceLink users.

The IDEAS HMD operated Android (custom) application has three main sub-applications that are providing functionality for team communication: "Calls", environmental monitoring and warnings: "Sensors", and guidance and navigation in the procedural environment: "Work". Each sub-application is developed and has dedicated functions in the framework of specific software and operational NASA KSC standards. Functional concept of the IDEAS GUI software applications as displayed via the HMD is depicted on Figure 9 below.

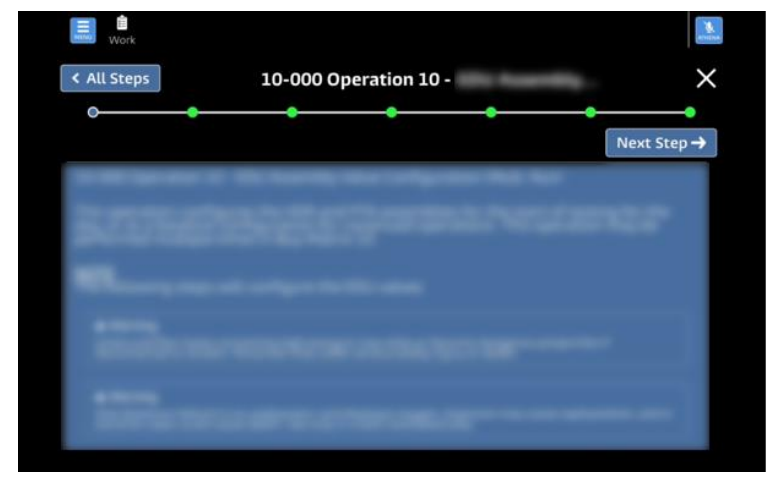

Figure 9: Screenshot of the IDEAS display interface. Blue field contains documentation, procedures, visual navigation etc. Top switches allow virtual access to the team database, procedures, sensors' data etc.

\section{Proposed hardware architecture - ergonomics}

The diversity of wearability options was studied, generating over 50 concepts out of which the main two ergonomic options were chosen and only one was implemented. A wearable head band capable of carrying the distributed IDEAS hardware with additional components on belt, chest or shoulder was one option, the second, implemented option, considered is integrated directly onto a hardhat. This option seemed easier to implement using COTS parts. Both digital and physical models (ergonomic models) have been developed to perform ergonomic tests in virtual and real environments.
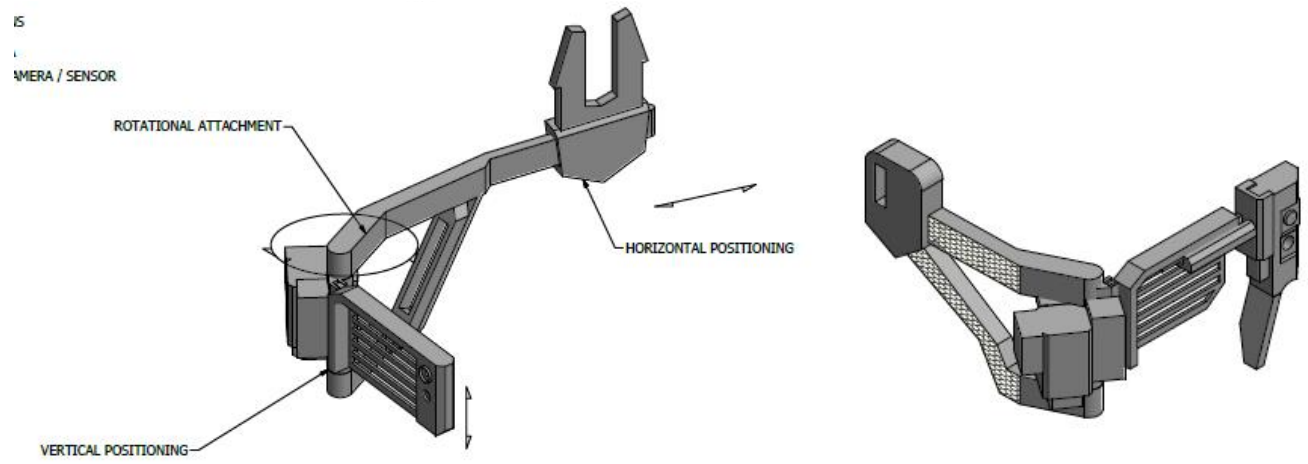

Figure 10: Two examples of early ergonomic monocle testers for hardhat and custom head mount.

A variety of ergonomic test devices were developed during the two years of the IDEAS project in order to clarify all nuances of users requirements related to comfort, operability, and usefulness in a variety of scenarios. One of the options considered a side projection monocle (see Figure 10) that could be superposed over users' glasses. This option 
was used for electronics development and was surpassed by a top projection position that has been selected for final product architecture configuration (Figure 11) as the ergonomically most effective option.

To ensure required performance of all sensors and HUD on the system and keep the system as light as possible, it was physically divided in three parts located on head (Figure 11), helmet or shoulder (backpack strap) and back or waist (backpack or belt). While the signal processing was performed by the helmet or shoulder GPU/CPU the power pack was placed inside the backpack or on the belt.

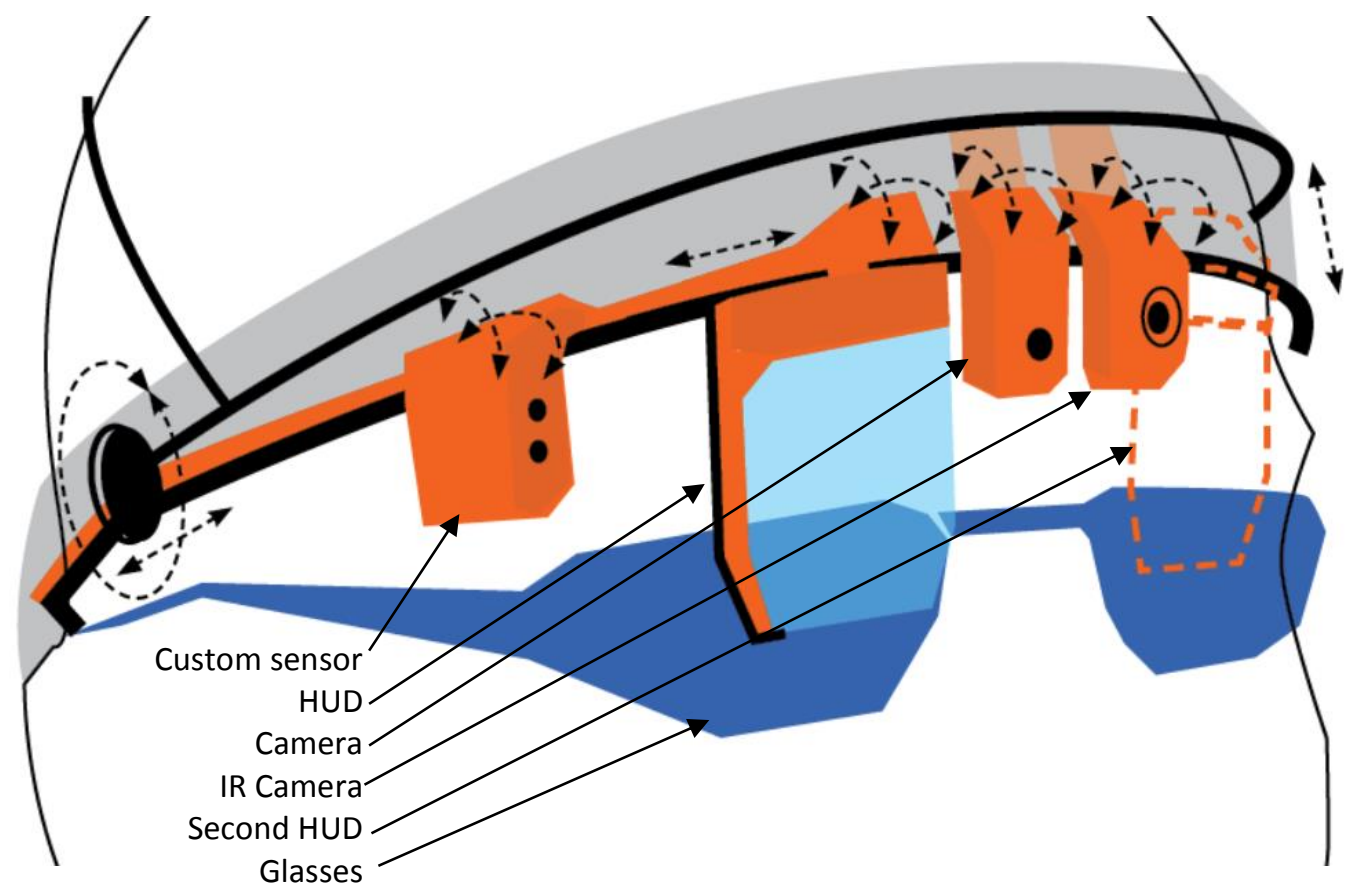

Figure 11: Final hardware architecture sketch based on exchangeable modules concept allowing prescription or PPE glasses (blue) and not limiting peripheral vision.

\section{Proposed hardware system architecture - electronics}

The Single Board Computer (SBC) running the Android OS is attached to the waist to minimize weight on the head. All computing is completed from the SBC. The display output and camera inputs are connected through a wire harness from the SBC to the head unit. The IR camera interfaces through USB while the RGB camera connects through a MIPI-CSI interface. The HUD is an LCOS glass display with an LVDS connection. The interface runs from an HDMI to LVDS converter daughter board attached to the SBC to a small light weight display controller attached to the head unit.

On power up, the Android SBC initializes the display controller and enables the RGB and IR camera. Once the boot up process finishes, the display turns on with the Android OS screen displayed to the user on the HUD.

\section{COTS Hardware compliance with IDEAS}

The continuous COTS components research by the hardware team was based on design requirements as well as financial and geographical availability. The components were tested as individual units for their applicability to the IDEAS system. Selection of the most important part i.e., HMD was constrained by the cost, and most importantly by the optical engine and collimator design with specific focus, field of view, contrast and other parameters. It has been identified that there is no perfect IDEAS hardware HMD solution during the prototype development (year 2016) and hence the design requirements had to be weakened in order to successfully complete the project. 


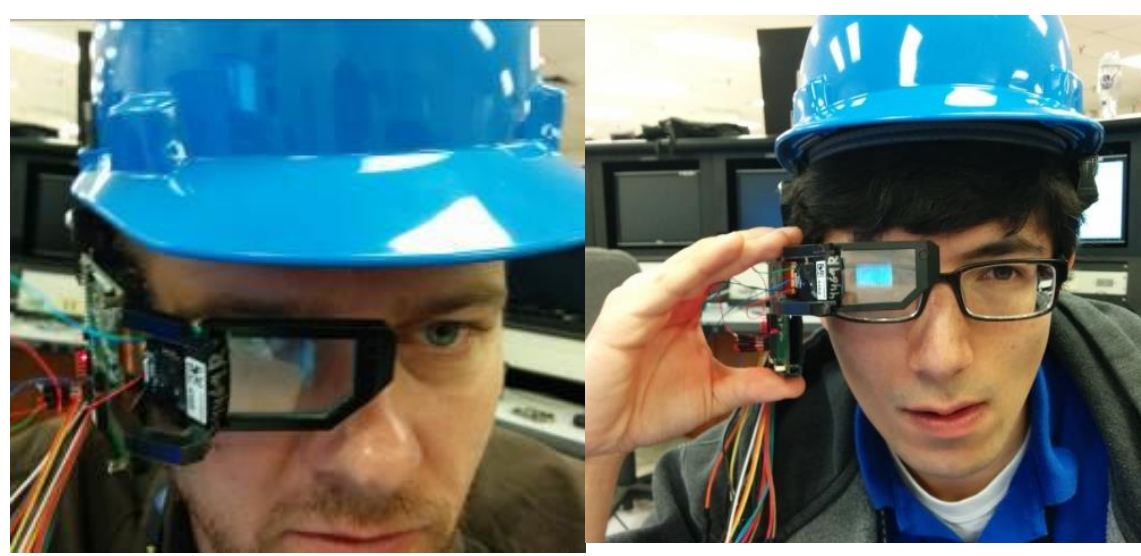

Figure 12: Early version collimator usability tests (optical engine: OE-32 Optical engine with 720p resolution (1366x768). I believe power was about $3 \mathrm{w}, \sim 35$ degree FOV, 4000 nit brightness) with and without glasses using temporal bone (side) position and hardhat as the subsystems wearability support. Tests concluded that side position of the projector will never comply with individual anthropometry and ergonomics requirements.

\section{HMD Architecture options}

While the software architecture has been selected in the initial stage of the project based on supportability, maintainability, documents compatibility, open source access and security requirements, the hardware architecture was considering a number of options based on existing fully integrated end user COTS products as well as COTS electronics components. The four options were considered until the mid of the project while priority decisions stemmed from COTS electronics components readily available and individual qualitative ergonomics tests. The following options were considered and are categorized in Table 1.

Table 1: HMD architecture options considered and IDEAS preferred architecture qualitative comparison.

\begin{tabular}{|c|c|c|c|c|}
\hline \# & $\begin{array}{l}\text { Projector position } \\
\text { Mono / Stereo vision }\end{array}$ & $\begin{array}{l}\text { Applicable } \\
\text { integrated COTS } \\
\text { option }\end{array}$ & Constraints & Benefits \\
\hline 1 & $\begin{array}{l}\text { Side } \\
\text { Stereo }\end{array}$ & $\begin{array}{l}\text { Epson Moverio } \\
\text { (Android), Atheer } \\
\text { AiR (Android) }\end{array}$ & $\begin{array}{l}\text { Peripheral vision limits, } \\
\text { difficulty or invalid for } \\
\text { prescription or other } \\
\text { glasses, limited } \\
\text { ergonomics, see } \\
\text { through limits field of } \\
\text { view, HW functions } \\
\text { limitations / no IR out } \\
\text { of the box }\end{array}$ & $\begin{array}{l}\text { Light \& SW user } \\
\text { friendly, right level of } \\
\text { system complexity, } \\
\text { highly integrated }\end{array}$ \\
\hline 2 & $\begin{array}{l}\text { Side } \\
\text { Mono }\end{array}$ & $\begin{array}{l}\text { Google Glass } \\
\text { (Android) }\end{array}$ & $\begin{array}{l}\text { Minor peripheral vision } \\
\text { limits, difficulty with } \\
\text { prescription or other } \\
\text { glasses, sensors } \\
\text { limitations, HW } \\
\text { functions limitations / } \\
\text { no IR out of the box, } \\
\text { sensors modularity }\end{array}$ & $\begin{array}{l}\text { Field of view } \\
\text { superposition, ultra- } \\
\text { light, applicable with } \\
\text { hardhat, highly } \\
\text { integrated }\end{array}$ \\
\hline
\end{tabular}




\begin{tabular}{|l|l|l|l|l|}
\hline 3 & $\begin{array}{l}\text { Top } \\
\text { Stereo }\end{array}$ & $\begin{array}{l}\text { Microsoft } \\
\text { HoloLens } \\
\text { (Windows), ODG } \\
\text { R-7 (Android) }\end{array}$ & $\begin{array}{l}\text { Mass and power, no } \\
\text { hardhat version, can be } \\
\text { too complex, larger, no } \\
\text { IR out of the box, no } \\
\text { sensor modularity, } \\
\text { vision and peripheral } \\
\text { vision limitations }\end{array}$ & $\begin{array}{l}\text { High level ergonomics, } \\
\text { high performance, } \\
\text { highly integrated }\end{array}$ \\
\hline $\begin{array}{l}\text { Top } \\
\text { Mono / expandable to stereo }\end{array}$ & $\begin{array}{l}\text { Minimum COTS } \\
\text { components available, } \\
\text { high level of HW / SW } \\
\text { development required, } \\
\text { complex signal } \\
\text { processing - distributed }\end{array}$ & $\begin{array}{l}\text { Excellent ergonomics, } \\
\text { applicable for users } \\
\text { with glasses, hardhat, } \\
\text { modular (1 or two } \\
\text { displays, 0-3 cameras), } \\
\text { 6deg adjustable } \\
\text { position within user's } \\
\text { field of view }\end{array}$ \\
\hline
\end{tabular}

The IDEAS configuration of HMD fulfilled the primary requirements on ease of use, mounting, modularity, user's ergonomics use with glasses, adjustability in the field of view (positioning sideways and up or down). The IDEAS system including HMD module, optical camera and IR module prototype have been successfully tested in laboratory environment.

\title{
XI. Conclusion
}

Organization design

One of the first conclusions of the project is that COTS software maturity far exceeds hardware maturity in terms of the state of the art. The IDEAS team confirmed the most suitable and readily available COTS hardware components are not sufficient for the development of integrated COTS hardware that could effectively be used in practical settings - meaning: IDEAS product as a fully functional robust product would require also custom electronics, optics, computer development, optimization of HMD COTS components, wearable computer parts, sensors, etc. which in practice would mean an order of magnitude higher budget. The software though was solely dependent on hardware compatibility and did not pose any serious risk after careful tradeoff analyses on software platforms and software architecture selection. This environment forced the team to perform ad-hoc research sessions during the design process and gather state of the art intelligence more frequently than originally expected.

System design

The IDEAS project was successfully completed and presented in January 2017 at the conclusive meeting with stakeholders. The team accomplished development of a functional hardware and software prototype as presented by the system architecture on Figure 8 and the product sketch on Figure 11. The project demonstrated that detailed user and environmental function analysis is necessary to understand the product configuration. High cost and complexity of the modular system design (Figure 11) led to the selection of COTS integrated hardware and the main design requirements were adapted.

The IDEAS project has been further split on the hardware part that has been suspended and the software part that has been further developed for a selected fully integrated hardware COTS product.

\section{Acknowledgments}

This project was selected and executed in scope of the NASA Space Technology Mission Directorate.

\author{
The IDEAS team: \\ Allan Villorin \\ Daniel Strohschein \\ David Miranda \\ Delvin VanNorman \\ Gavin Roberts \\ Hector Pagan \\ Jake Hochstadt \\ Joshua Santora
}

Copyright ( 2017 by Ondrej Doule, Ph.D., David Miranda and Jake Hochstadt Published by the American Institute of Aeronautics and Astronautics, Inc., with permission. 
AIAA Space Forum 2017, September 12-14, Orlando, FL

\author{
Julianna Wood \\ Kelvin Ruiz \\ Matt Eagle \\ Michael McDonough \\ Ondrej Doule \\ Paulus Hilt \\ IDEAS interns: \\ Andrzej Jackowski \\ Beth Dube \\ Daniel Wells \\ Derek Daniels \\ Hannah Misenar \\ Joseph Torkaman \\ Kyle Otsuka \\ Rabab Alrayes \\ Roy Ramirez
}

\title{
References
}

\footnotetext{
${ }^{1}$ Friedrich, W., 2002, “ARVIKA-Augmented Reality for Development, Production and Service”, Proceedings of the International Symposium on Mixed and Augmented Reality (ISMAR'02), Siemens AG, Automation and Drives, Advanced Technologies and Standards, Germany.

${ }^{2}$ Lukowicz, P., Timm-Giel, A., Lawo, M., Herzog, O., 2007, “Wearable Computing”, WearIT@work: Toward Real-World Industrial Wearable Computing, Wearable Computing, Starner, Thad E.,IEEE Computer Society, 1536$1268 / 07$

${ }^{3}$ Vincenzi, D.A., Deaton, J.E., Buker, T.J., Blickensderfer, E.L., Williams, B., Pray, R., 2011, "Mitigation of System Latency in Next Generation Helmet Mounted Display Systems", Proceedings of Human Factors and Ergonomics Society, $55^{\text {th }}$ annual meeting.

${ }^{4}$ Melzer, J., 2015, “Head-Mounted Displays,” Digital Avionics Handbook, Third Edition, edited by Cary R.Spitzer, Uma Ferrell, Thomas Ferrell, CRC Press, Taylor \& Francis Group, Boca Raton, London, New York

${ }^{5}$ Doule, O., Poulet, L., 2014, "Ergonomy of Head Mounted Displays Inside Analog Spacesuit - Mars Analog Extravehicular Activities", AIAA SPACE 2014 Conference and Exposition, AIAA SPACE Forum, (AIAA 20144406), Available Online: https://doi.org/10.2514/6.2014-4406 [Accessed July 6, 2017].

${ }^{6}$ Boy, G.A., 1997, "Cognitive function analysis: an example of human centered re-design of a flight management system", Proceedings of the $13^{\text {th }}$ Triennial Congress of the International Ergonomics Association, June 29-July 4, 1997, Tampere, Finland.
} 